\title{
Implicit and Explicit Aspect Extraction in Financial Microblogs
}

\author{
Thomas Gaillat, Bernardo Stearns, Gopal Sridhar, \\ Ross McDermott, Manel Zarrouk \\ Insight Centre for Data Analytics NUI Galway \\ firstname.surnamedinsight-centre.org
}

Brian Davis

Maynooth University

brian.davis@mu.ie

\begin{abstract}
This paper focuses on aspect extraction which is a sub-task of Aspect-based Sentiment Analysis. The goal is to report an extraction method of financial aspects in microblog messages. Our approach uses a stock-investment taxonomy for the identification of explicit and implicit aspects. We compare supervised and unsupervised methods to assign predefined categories at message level. Results on 7 aspect classes show 0.71 accuracy, while the 32 class classification gives 0.82 accuracy for messages containing explicit aspects and 0.35 for implicit aspects.
\end{abstract}

\section{Introduction}

Sentiment Analysis (SA) in the financial domain has shown a growing interest in recent years. Acquiring an insight into the public opinion of relevant and valuable economic signals can give a competitive edge and allow more informed investment decisions to be executed. Microblog platforms such as Twitter and StockTwits, are central to determining these economic signals (Bollen et al., 2011; Zhang et al., 2011). Investors share their opinions about stocks, companies and products, and these contents are valuable for whomever is interested in predicting market trends. Research in the area of SA tries to shed some light on this problem. Its purpose is to identify opinions and sentiments that are directed towards entities such as stocks and companies or towards the attributes, or aspects, of these entities.

The authors are involved in SSIX ${ }^{1}$ (Davis et al., 2016), a project focused on SA in financial markets. It currently offers sentiment scores for

\footnotetext{
${ }^{1}$ Social Sentiment IndeX is a platform dedicated to SA in financial microblogs. Available at https://ssix-project.eu/
}

stocks and companies and intends to provide finergrained SA by including aspects. In order to conduct Aspect-Based SA in this project, the first step is to identify aspects in microblog messages, which is the focus of this paper.

As stated in SemEval-2015, the problem in Aspect-based SA can be divided into three subtasks, i.e. aspect category identification, Opinion Target Expression (OTE) extraction and sentiment polarity assignment (Pontiki et al., 2015). In this paper, we focus on the first sub-task of aspect category assignment. There have been two types of approaches to conduct this subtask. In the first type, aspect words are extracted and clustered (Qiu et al., 2011; Chen and Liu, 2014; Shu et al., 2016; Poria et al., 2016). In the second type, predefined aspects categories are assigned to entity-attribute pairs at sentence level (Pontiki et al., 2015). The first type of approaches targets explicit aspects while the second one also includes implicit aspects, i.e. aspects that are not explicitly mentioned in the text strings (Liu, 2012, p. 77). Using predefined aspects corresponds to the project requirements but most approaches deal with hotel, restaurant and product-related data. To the best of our knowledge none of them use a corpus of annotated aspects in the financial domain.

We present a method that focuses on the aspect category identification of implicit and explicit aspects. The originality of our work is to evaluate different aspect category identification approaches based on a predefined taxonomy of stock-investment aspects. Work is carried out on a limited data set with a view to expanding it should results be satisfactory. Our approach relies on using a corpus of annotated messages to build several types of models based on distributional semantics and supervised learning methods. Also original is that our work focuses on the stockinvestment domain as it is to be added to the SSIX 
platform. The remainder of this paper is divided as follows. Section 2 covers related work. Section 3 gives details about the corpus that was used. In Section 4 the different models are described. Results are presented in Section 5 , followed by the conclusion in Section 6

\section{Related Work}

Available methods in aspect category identification can be divided into supervised and unsupervised approaches. Unsupervised approaches include a number of lexicon-based strategies relying on i) frequency measures used with association measures such as Point-wise Mutual Information (PMI) to link words with lexicon entries (Popescu and Etzioni, 2005; Long et al., 2010), ii) syntactic relations to relate core sentiment words, expressed by adjectives, to target aspect words expressed by nouns (Liu et al., 2016; Fang and Huang, 2012; Jo and Oh, 2011; Brody and Elhadad, 2010; Chen and Liu, 2014), and iii) on word association measures for topic extractions and clustering methods (Fang and Huang, 2012; Jo and Oh, 2011; Brody and Elhadad, 2010; Chen and Liu, 2014). All these methods rely on lexicons to search for explicit words linked to aspects.

Supervised approaches rely on Machine Learning (ML) algorithms that are trained on classified instances of aspects prior to performing classification of new instances. Many studies have proposed different types of Conditional Random Fields (CRF) models (Jakob and Gurevych, 2010; Mitchell et al., 2013; Shu et al., 2016; Cruz et al., 2014; Poria et al., 2016) that distinguish aspects from non-aspects in text sequences. In parallel, other methods apply aspect category identification on the basis of predefined aspects linked to Entity (E) and Attribute (A) pairs (Pontiki et al., 2015, 2014). The current SemEval framework requires the extraction of explicit mentions of $\mathrm{E}$ and of all mentions of A (implicit and explicit)(Pontiki et al., 2015).

With respect to the implicit / explicit distinction, traditional approaches have focused on explicit aspects (Liu et al., 2016; Schouten et al., 2018), hence relying on word occurrences to determine aspects. Other, more novel, methods have focused on identifying implicitly-referred-to aspects (Pontiki et al., 2015). (Dosoula et al., 2016) developed an implicit feature algorithm that uses cooccurrences to assign implicit aspects at sentence level in online restaurant reviews.

Our framework is similar to SemEval-2015 Task 12 (Pontiki et al., 2015) insofar as we used predefined categories of aspects (A) for stocks considered as entities (E). Likewise, our approach includes the extraction of aspects that are not necessarily mentioned in messages. The difference is that we use a two-level aspect taxonomy for coarse and fine-grained characterization, which gives 32 fine-grained classes as opposed to the 9 classes of the laptop data set of SE-2015 task 12 for instance. We also conduct category identification at message level without creating E/A pairs. For the requirements of the project, we use a specific financial aspect taxonomy. Albeit applied to a different domain, results show higher or equivalent F1-Scores depending on the granularity.

\section{Corpus}

The approach relies on a corpus of messages specialised in stock trading ${ }^{2}$. Microblog messages were posted by stock traders who share investment ideas and intelligence. The data set is described in Table 1.

\begin{tabular}{ll}
\hline Aspect type & Number of messages \\
\hline All types & 368 \\
Implicit aspects & 218 \\
Explicit aspects & 150 \\
\hline
\end{tabular}

Table 1: Number of implicit and explicit messages in the data set

\subsection{Taxonomy of Stock-Investment Aspects}

As a preliminary step to aspect identification, a financial expert defined a taxonomy of trading aspects (See Appendix). They were grouped on the basis of hypo/hypernym relations following a general to more specific hierarchy. The final taxonomy consists in an aspect class dominating an aspect sub-class. No related terms, nor synonyms, were added to these subclasses. There are 7 aspect classes, e.g. User Action, Asset Direction and 32 aspect subclasses, e.g. User Action $>$ Buying Intention. Aspect classes do not include the same number of subclasses. For instance, the User Action class includes 5 aspect subclasses while the User Outlook class includes 2 aspect subclasses. The

\footnotetext{
${ }^{2}$ The dataset is available at https://bitbucket.org/ssixproject/stock-investment-aspect-extraction
} 
taxonomy is used i) to compute the semantic relatedness between taxonomy labels and textual candidates (DSM approach. See Section 4.1) and ii) to relate message features with taxonomy classes (Supervised-learning approach. See Section 4.2.

\subsection{Annotation Scheme}

The messages were manually classified by one financial expert according to the afore-mentioned taxonomy by matching aspect classes and subclasses with messages. Annotation includes the message ID and the OTE that substantiates the selected class. The following example is a JSONtype extract of the first message classified as User Outlook $>$ Negative Outlook.

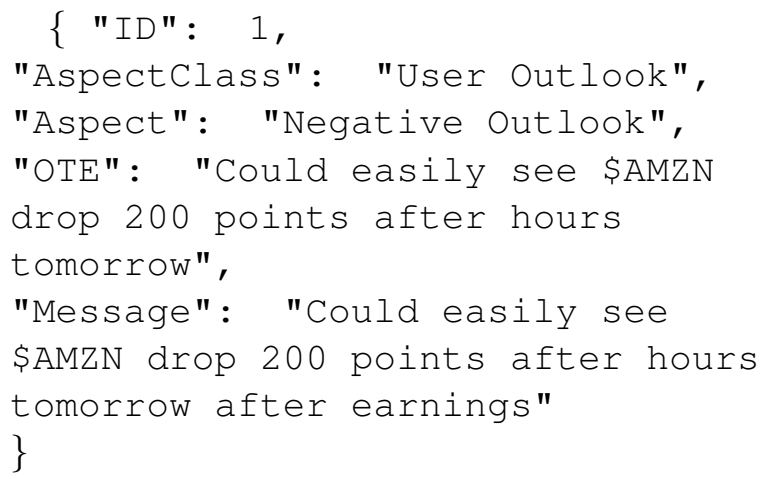

\section{Building a Classification Model}

This section focuses on the method used to build different models for the aspect extraction task. The task of the classifier is to assign (i) aspect classes and (ii) subclasses to messages. In this section, we present the two approaches. The first one applies a distributional semantics model, while the second one is based on several Machine Learning algorithms.

\subsection{Distributional Semantics Model (DSM)}

This approach relies on word embeddings for the computation of semantic relatedness with Word2vec (Mikolov et al., 2013). Word embeddings fall in the category of distributional semantics methods in which the meaning of a word is related to the distribution of words around it ( $\mathrm{Ju}$ rafsky and Martin, 2009, p.659-665).

Word2vec, in its skip-gram architecture, is such a model and was trained on the Google news corpus. The vector values are the weights computed by the hidden layer of a Neural Network trained on a corpus. The Word2vec skip-gram model allows to find words that appear frequently together, and infrequently in other contexts (Mikolov et al.
2013).

The task of identifying aspects can be formulated as mapping textual elements of messages to their most related aspect class label in the taxonomy. There are two steps: extracting candidates and computing relatedness with the classes.

\subsubsection{Extracting Candidates}

After preprocessing (tokenisation and Part-ofSpeech (POS) tagging) The extraction of candidates relies on rule-based heuristics using morphosyntactic patterns to select relevant Noun Phrases and Verb Phrases including modifiers such as adverbs, adjectives and present participles. The purpose is to capture fine-grained senses of these phrases. Example (1) illustrates the extraction of the item declining revenue.

1) $\$ M C D$ with declining revenue for a good while

In example (1) only declining revenue is extracted. This segment is semantically relevant for the classification as Revenue Down, while the remainder of the NP does not procure any information regarding the type of aspect.

\subsubsection{Computing Semantic Relatedness}

Computing semantic relatedness consists of comparing vectors of candidates with vectors of aspect subclasses. First, multi-word candidates or labels are combined into single vectors to obtain pairs of candidate-aspect vectors. The method is the sum of the vectors of multi-word expressions. To compute relatedness between vectors, we use the Indra implementation (Freitas et al., 2016) of the cosine similarity metric. The system computes cosine similarity for all possible pairwise combinations of tokens in each message. We retain the pair with the highest score.

\subsection{Supervised Learning Models}

This approach relies on training several machinelearning models. Building the classifier consists in a multi-class supervised classification task.

\subsubsection{Feature Engineering}

After preprocessing (tokenisation, accent removal, lower-casing and POS tagging), messages were converted into vectors including the following features:

- Bag of Words (BoW) - They are used to create a numerical representation of the vocabulary of messages. We use three types of statis- 
tics (binary count, frequency count and tf-idf) applied on n-gram clusters.

- Part of Speech - PoS are used to create a numerical representation of the POS present in each message. This representation is based on the Penn Treebank POS tagset(Marcus et al., 1993).

- Numericals - These are used to create a representation of financial values mentioned in the messages such as percentages, ratios, stock prices and amounts (e.g. \$55).

- Predicted sentiment of entity- The sentiment predicted ${ }^{3}$ on the financial entities included in the messages that may contain aspects. It is a continuous value on a $[-1 ; 1]$ range.

\subsubsection{Machine-Learning Algorithms and Optimization}

A number of Machine Learning Python-based models were tested. Two methods are based on decision trees with XGboost (Chen and Guestrin, 2016) and Random Forests (Breiman, 2001). We also used Support Vector Machines (Vapnik, 2000) and Conditional Random Fields (Lafferty et al., 2001). Each of these methods use the same vector representation created in the feature engineering phase.

In order to find the best hyper-parameters for the tested models, we used the Particle Swarm Optimization (PSO) method. This method was appropriate due to the fact that hyper-parameters are numbers, mostly in a continuous space. PSO (Kennedy and Eberhart, 1995) was applied using 100 particles (specific hyper-parameter configurations) during 100 iterations, using same weights for velocity, particle best and global best. For each particle position, the average accuracy in 10 -fold cross validation was calculated.

\subsection{Model Selection, Validation and Evaluation}

Choosing the best classifier is done in two stages. Firstly, a model selection procedure helps select the best model among the DSM and ML models. All models were tested with 10 -fold crossvalidation whereby the dataset is divided in ten parts. Each part is used as a test set once in the ten

\footnotetext{
${ }^{3}$ with the use of the SSIX FinSentiA Sentiment Analyser (Gaillat et al., 2018)
}

iterations of the process. Secondly, the selected model is validated by using the leave-one-out option, meaning that the training is conducted on all instances except one. The process is repeated until all instances have been used as a test instance.

In the model selection stage we computed global accuracy for 32 classes. In the validation stage, we used F1-Score for 7 and 32 classes to measure the effects of the coarse and fine-grained annotation levels. The annotated corpus described in Section 3 was used for training and testing. In the DSM approach, 172 initially annotated messages were used as test set.

\section{Results and Discussion}

In the model selection stage all of the approaches show different results as shown in Table 2 .

\begin{tabular}{rll}
\hline Model & Accuracy & $\begin{array}{l}\text { Standard } \\
\text { deviation }\end{array}$ \\
\hline $\begin{array}{r}\text { DSM (baseline) } \\
\text { ML Methods }\end{array}$ & 0.425 & - \\
Xgboost & 0.5689 & 0.046 \\
Random Forest & 0.5435 & 0.038 \\
SVC & 0.449 & 0.027 \\
CRF & 0.431 & 0.052 \\
\hline
\end{tabular}

Table 2: Model selection stage: Accuracy for each model for the 32 aspect classification task

Xgboost was selected and validation showed results (see Table 3 ) in line with the best scores obtained in SemEval-2015 Task 12.

Table 4 shows the accuracy for message classification according to the implicit or explicit nature of the 32 aspects. The distinction between implicit and explicit aspect messages shows that explicit aspects are well classified while implicit aspects are only correctly handled in about $35 \%$ of cases. This suggests that the classifier lacks significant features to identify implicit aspects. The size of the data set appears to be a limitation but the size of sentences may also impair the classifier by adding noise to the data. Using aspect-relevant OTEs as a BoW feature could help address this point.

\section{Conclusion and Future Work}

In this paper, we have reported on a series of experiments in the domain of Aspect Extraction. The experiments focused on the sub-task of aspect cat- 


\begin{tabular}{lllll}
\hline Model & Acc & F1-Score & P & R \\
\hline $\begin{array}{l}\text { Xgboost } \\
\text { (32 classes) }\end{array}$ & 0.565 & 0.49 & 0.52 & 0.49 \\
$\begin{array}{l}\text { Xgboost } \\
\text { (7 classes) }\end{array}$ & 0.712 & 0.71 & 0.70 & 0.71 \\
\hline
\end{tabular}

Table 3: Model validation stage: Accuracy, F1Score, Precision (P) and Recall (R) for the 32 and 7 aspect classification task

\begin{tabular}{lllll}
\hline Aspects & Acc & F1-Score & P & R \\
\hline Implicit & 0.351 & 0.32 & 0.28 & 0.28 \\
Explicit & 0.826 & 0.8 & 0.84 & 0.8 \\
\hline
\end{tabular}

Table 4: Accuracy according to messages including 32 implicit and explicit aspects

egory identification in the domain of stock investments. A taxonomy was used to identify predefined aspects in microblog messages. A distributional semantics model and several supervised learning methods were used for the task.

Results show that explicit aspect identification performs well, but implicit aspect identification remains an issue that can be tackled with larger data set and improved feature engineering. Despite the size of the training data set, results suggest that more efforts can be invested in the development of a larger data set.

\section{Appendix}

Taxonomy of stock-investment aspects

- User Action

- Buying Intention

- Selling Intention

- Bought

- Sold

- Shorting

- User Outlook

- Positive Outlook

- Negative Outlook

- Insider Activity

- Insider Selling

- Insider Buying

- Asset Direction

- Moving Higher
- Moving Lower

- Breakout

- New High

- Trending Higher

- Trending Lower

- Trending Sideways

- Asset Behaviour

- Oversold

- Overbought

- Overvalued

- Undervalued

- Short Squeeze

- Selling Pressure

- Financial Results

- Earnings Beat

- Earnings Miss

- Revenue Up

- Revenue Down

- Profit Warning

- Analyst Ratings

- Buy Recommendation

- Sell Recommendation

- Rating Upgrade

- Rating Downgrade

\section{References}

Johan Bollen, Huina Mao, and Xiao-Jun Zeng. 2011. Twitter mood predicts the stock market. Journal of Computational Science, 2(1):1-8.

Leo Breiman. 2001. Random Forests. Machine Learning, 45(1):5-32.

Samuel Brody and Noemie Elhadad. 2010. An Unsupervised Aspect-sentiment Model for Online Reviews. In Human Language Technologies: The 2010 Annual Conference of the North American Chapter of the Association for Computational Linguistics, HLT '10, pages 804-812, Stroudsburg, PA, USA. Association for Computational Linguistics.

Tianqi Chen and Carlos Guestrin. 2016. XGBoost: A Scalable Tree Boosting System. In Proceedings of the 22Nd ACM SIGKDD International Conference on Knowledge Discovery and Data Mining, KDD '16, pages 785-794, New York, NY, USA. ACM. 
Zhiyuan Chen and Bing Liu. 2014. Topic Modeling Using Topics from Many Domains, Lifelong Learning and Big Data. In Proceedings of the 31st International Conference on International Conference on Machine Learning - Volume 32, ICML'14, pages II-703-II-711, Beijing, China. JMLR.org.

Ivan Cruz, Alexander F. Gelbukh, and Grigori Sidorov. 2014. Implicit Aspect Indicator Extraction for Aspect based Opinion Mining. Int. J. Comput. Linguistics Appl., 5:135-152.

Brian Davis, Keith Cortis, Laurentiu Vasiliu, Adamantios Koumpis, Ross Mcdermott, and Siegfried Handschuh. 2016. Social Sentiment Indices Powered by X-Scores. In ALLDATA 2016, The Second International Conference on Big Data, Small Data, Linked Data and Open Data, Lisbon, Portugal. International Academy, Research, and Industry Association ( IARIA ).

Nikoleta Dosoula, Roel Griep, Rick den Ridder, Rick Slangen, Ruud van Luijk, Kim Schouten, and Flavius Frasincar. 2016. Sentiment Analysis of Multiple Implicit Features per Sentence in Consumer Review Data. In Databases and Information Systems (DB\&IS), Riga, Latvia. Springer.

Lei Fang and Minlie Huang. 2012. Fine Granular Aspect Analysis Using Latent Structural Models. In Proceedings of the 50th Annual Meeting of the Association for Computational Linguistics: Short Papers - Volume 2, ACL '12, pages 333-337, Stroudsburg, PA, USA. Association for Computational Linguistics.

André Freitas, Siamak Barzegar, Juliano Efson Sales, Siegfried Handschuh, and Brian Davis. 2016. Semantic Relatedness for All (Languages): A Comparative Analysis of Multilingual Semantic Relatedness Using Machine Translation. In Eva Blomqvist, Paolo Ciancarini, Francesco Poggi, and Fabio Vitali, editors, Knowledge Engineering and Knowledge Management: 20th International Conference, EKAW 2016, Bologna, Italy, November 19-23, 2016, Proceedings, pages 212-222. Springer International Publishing, Cham.

Thomas Gaillat, Annanda Sousa, Manel Zarrouk, and Brian, Davis. 2018. FinSentiA: Sentiment Analysis in English Financial Microblogs. In Proceedings of the TALN-CORIA 2018, Rennes, France. Revue TAL.

Niklas Jakob and Iryna Gurevych. 2010. Extracting Opinion Targets in a Single- and Cross-domain Setting with Conditional Random Fields. In Proceedings of the 2010 Conference on Empirical Methods in Natural Language Processing, EMNLP' 10, pages 1035-1045, Stroudsburg, PA, USA. Association for Computational Linguistics.

Yohan Jo and Alice H. Oh. 2011. Aspect and Sentiment Unification Model for Online Review Analysis. In Proceedings of the Fourth ACM International Conference on Web Search and Data Mining,
WSDM '11, pages 815-824, New York, NY, USA. ACM.

Daniel Jurafsky and James H. Martin. 2009. Speech and Language Processing (2nd Edition). PrenticeHall, Inc., Upper Saddle River, NJ, USA.

James Kennedy and Russel Eberhart. 1995. Particle swarm optimization. In , IEEE International Conference on Neural Networks, 1995. Proceedings, volume 4, pages 1942-1948 vol.4.

John D. Lafferty, Andrew McCallum, and Fernando C. N. Pereira. 2001. Conditional Random Fields: Probabilistic Models for Segmenting and Labeling Sequence Data. In Proceedings of the Eighteenth International Conference on Machine Learning, ICML '01, pages 282-289, San Francisco, CA, USA. Morgan Kaufmann Publishers Inc.

Bing Liu. 2012. Sentiment Analysis and Opinion Mining. Morgan \& Claypool Publishers, San Rafael, Calif.

Qian Liu, Bing Liu, Yuanlin Zhang, Doo Soon Kim, and Zhiqiang Gao. 2016. Improving Opinion Aspect Extraction Using Semantic Similarity and Aspect Associations. In Thirtieth AAAI Conference on Artificial Intelligence, Phoenix, Arizona USA. AAAI Press.

Chong Long, Jie Zhang, and Xiaoyan Zhu. 2010. A Review Selection Approach for Accurate Feature Rating Estimation. In Proceedings of the 23rd International Conference on Computational Linguistics: Posters, COLING '10, pages 766-774, Stroudsburg, PA, USA. Association for Computational Linguistics.

Mitchell P. Marcus, Mary Ann Marcinkiewicz, and Beatrice Santorini. 1993. Building a Large Annotated Corpus of English: The Penn Treebank. Computational Linguistics, 19(2):313-330.

Tomas Mikolov, Kai Chen, Greg Corrado, and Jeffrey Dean. 2013. Efficient Estimation of Word Representations in Vector Space. ArXiv: 1301.3781.

Margaret Mitchell, Jacqueline Aguilar, Theresa Wilson, and Benjamin Van Durme. 2013. Open Domain Targeted Sentiment. In Proceedings of the 2013 Conference on Empirical Methods in Natural Language Processing, pages 1643-1654, Seattle, Washington, USA. ACL.

Maria Pontiki, Dimitris Galanis, Haris Papageorgiou, Suresh Manandhar, and Ion Androutsopoulos. 2015. SemEval-2015 Task 12: Aspect Based Sentiment Analysis. In Proceedings of the 9th International Workshop on Semantic Evaluation (SemEval 2015), pages 486-495, Denver, USA. The Association for Computational Linguistics.

Maria Pontiki, Dimitris Galanis, John Pavlopoulos, Harris Papageorgiou, Ion Androutsopoulos, and Suresh Manandhar. 2014. SemEval-2014 Task 4: 
Aspect Based Sentiment Analysis. In Proceedings of the 8th International Workshop on Semantic Evaluation (SemEval 2014), pages 27-35, Dublin, Ireland. Association for Computational Linguistics and Dublin City University.

Ana-Maria Popescu and Oren Etzioni. 2005. Extracting Product Features and Opinions from Reviews. In Proceedings of the Conference on Human Language Technology and Empirical Methods in Natural Language Processing, HLT '05, pages 339-346, Stroudsburg, PA, USA. Association for Computational Linguistics.

Soujanya Poria, Erik Cambria, and Alexander Gelbukh. 2016. Aspect extraction for opinion mining with a deep convolutional neural network. Knowledge-Based Systems, 108(Supplement C):4249.

Guang Qiu, Bing Liu, Jiajun Bu, and Chun Chen. 2011. Opinion Word Expansion and Target Extraction Through Double Propagation. Comput. Linguist., 37(1):9-27.

K. Schouten, O. van der Weijde, F. Frasincar, and R. Dekker. 2018. Supervised and Unsupervised Aspect Category Detection for Sentiment Analysis with Co-occurrence Data. IEEE Transactions on Cybernetics, 48(4):1263-1275.

Lei Shu, Bing Liu, Hu Xu, and Annice Kim. 2016. Supervised Opinion Aspect Extraction by Exploiting Past Extraction Results. CoRR, abs/1612.07940.

Vladimir Vapnik. 2000. The Nature of Statistical Learning Theory, 2 edition. Information Science and Statistics. Springer-Verlag, New York.

Xue Zhang, Hauke Fuehres, and Peter A. Gloor. 2011. Predicting Stock Market Indicators Through Twitter I hope it is not as bad as I fear. Procedia - Social and Behavioral Sciences, 26(Supplement C):55-62. 